\title{
A heterogeneous population model for contagious bovine pleuropneumonia transmission and control in pastoral communities of East Africa
}

\author{
J.C. Mariner ${ }^{\mathrm{a}, *}$, J. McDermott ${ }^{\mathrm{b}}$, J.A.P. Heesterbeek ${ }^{\mathrm{c}}$, \\ G. Thomson ${ }^{\mathrm{d}}$, P.L. Roeder ${ }^{\mathrm{e}}$, S.W. Martin ${ }^{\mathrm{f}}$ \\ ${ }^{a}$ RDP Livestock Services, PO Box 523, 3700 AM Zeist, The Netherlands \\ ${ }^{\mathrm{b}}$ ILRI, PO Box 30709, Nairobi, Kenya \\ ${ }^{\mathrm{c}}$ Faculty of Veterinary Medicine, Department of Farm Animal Health, University of Utrecht, \\ Yalelaan 7, 3584 CL Utrecht, The Netherlands \\ ${ }^{\mathrm{d}}$ Epidemiology Unit, Pan African Programme for the Control of Epizootics, \\ African Union/Interafrican Bureau for Animal Resources. \\ PO Box 30786, Nairobi, Kenya \\ ${ }^{\mathrm{e}}$ Animal Health Service, Food and Agriculture Organization of the United Nations, \\ Viale delle Terme di Caracalla, 00100 Rome \\ ${ }^{\mathrm{f}}$ Department of Population Medicine, University of Guelph, Guelph, Ont., Canada
}

Received 27 January 2005; received in revised form 21 August 2005; accepted 1 September 2005

\begin{abstract}
Pastoral cattle live in highly structured communities characterized by complex contact patterns. The present paper describes a spatially heterogeneous model for the transmission of contagious bovine pleuropneumonia (CBPP) developed specifically for pastoral communities of East Africa. The model is validated against serological data on the prevalence of CBPP infection in several communities of southern Sudan and against livestock owner information on community structure, livestock contact and cattle exchange. The model is used to assess the impact of alternative control strategies including mass and elective vaccination programmes, potential treatment regimes and the
\end{abstract}

\footnotetext{
* Corresponding author. Present address: Department of Environmental and Population Health, Tufts Cummings School of Veterinary Medicine, 200 Westboro Road, North Grafton, MA 01536, USA.

Tel.: +1 508839 5302/887 6762; fax: +1 5088397948 .

E-mail address: jeffreymariner@yahoo.com (J.C. Mariner).
} 
combination of vaccination and treatment in a single unified strategy. The results indicate that the eradication of CBPP using mass vaccination with currently available vaccines is unlikely to succeed. On the other hand, elective control programmes based on herd level vaccination, treatment of clinical cases or a combination of both vaccination and treatment enabled individual livestock owners to capture a large benefit in terms of reduced animal-level prevalence and mortality experience. The most promising intervention scenario was a programme which combined the vaccination of healthy animals with treatment of clinical cases.

(C) 2005 Published by Elsevier B.V.

Keywords: Contagious bovine pleuropneumonia; Modelling; Basic reproduction number; Participatory; Epidemiology; Policy-making

\section{Introduction}

Contagious bovine pleuropneumonia (CBPP) is a major constraint to cattle production in the key pastoral regions of East Africa. Many communities rank CBPP as 1 of their top 3 animal health concerns and are highly motivated to take action to reduce losses. The disease is transmitted by direct contact and only cattle and water buffaloes are known to be affected. The usual source of infection is actively infected cases and spread occurs through their movement. Although both asymptomatic sub-acute and chronic cases are common, their role in the spread of disease has not been objectively demonstrated (Masiga et al., 1996; Windsor and Masiga, 1977). The mobility, high inter-group contact rates and importance of livestock exchange in pastoral systems create special challenges for CBPP control programs. New paradigms for CBPP control that reflect the importance and reality of pastoral livelihoods are needed.

We have previously reported on a stochastic compartmental model for CBPP transmission in pastoral herds based upon a single homogeneous population (Mariner et al., 2006). The homogeneous model integrated epidemiological data on the transmission of CBPP from diverse sources for the study of CBPP dynamics and evaluation of control options. Local livestock owner knowledge on the patterns of CBPP in traditional communities was an important component of the analysis. Experimentation with the homogeneous model found the current mass vaccination approach to CBPP eradication was unlikely to be successful with available vaccines. On the other hand, the model indicated that elective vaccination or an effective treatment regime could reduce CBPP mortality leading to important private (herd-level) benefits.

Pastoral community structure is complex in social, spatial and temporal terms. The prevalence and impact of CBPP is known to vary widely between community groups within related areas and across ecological zones. This paper describes a spatially heterogeneous population model that was developed to take our initial model 1 step further and investigate the impact of pastoral community structure and contact patterns on the dynamics of CBPP transmission and control.

The spatially heterogeneous model was used to explore control options at the individual herd level taking into account contact with the surrounding pastoral community. There are 
four essential tools in livestock disease control. These are movement control, stamping out, vaccination and treatment. All act by reducing the effective reproductive number of the agent in the population, but not all are acceptable interventions in every situation (Hammond and Branagan, 1965). These 4 approaches will be examined in order to identify promising CBPP control strategies that are both effective and acceptable under today's pastoral conditions.

\section{Materials and methods}

\subsection{Pastoral community contact structure}

The participatory epidemiological techniques of semi-structured interviews, proportional piling (Catley et al., 2001, 2002), mapping and Venn diagramming (Mariner and Paskin, 2000) were used to collect information on the relative size, types of interactions and extent of contact between pastoral groups in the Boma area of southern Sudan and at Gibaso in northern Tanzania. In the semi-structured interviews, open-ended questions were used to identify tribal and clan groupings. In the proportional piling exercises, participants were asked to divide a pile of 100 counters to indicate the relative contact between different groups identified in the open-ended questions. In the visualization techniques, participatory mapping and Venn diagramming, the respondents where requested to draw diagrams that identified community groups, their spatial relationships and the degree of between-group social interaction.

\subsection{The heterogeneous population model}

The core model utilized in the construction of the multi-population model was a stochastic compartmental model containing six principal states: susceptible, vaccinated, exposed, infectious, persistently infected and recovered (SVEIQR). The structure of the core model, parameter definitions, the methods of parameter estimation, model validation and base-line experimentation have been described in a companion paper (Mariner et al., 2006). Parameter and compartment definitions together with default estimates and initial conditions are summarized in Table 1 . These values were used in all simulations unless otherwise stated. For those compartments not specified, an initial value of 0 was used.

The heterogeneous model consists of one structured pastoral population made up of three inter-linked sub-populations. This was considered to be an approximation of a pastoral community of three herds or cattle camps where the three sub-populations were herded separately at night but have contact during the day while on pasture and at watering points. The sub-population sizes could be set separately to model different community sizes. All model parameters were set globally. The initial conditions for the population size $(N)$ and the prevalence of infection in the exposed $(E)$, infectious $(I)$ and recovered $(R)$ states were set for each population.

The heterogeneous population model incorporated a population contact matrix $\left(\beta_{i j}\right)$ where within population effective contact rates were on the diagonal and between 
population contact rates were off the diagonal.

$$
\left[\begin{array}{lll}
\beta_{11} & \beta_{12} & \beta_{13} \\
\beta_{21} & \beta_{22} & \beta_{23} \\
\beta_{31} & \beta_{23} & \beta_{33}
\end{array}\right]
$$

The subscript $i$ denotes the target sub-population and $j$ the sub-population acting as the source of infection. When defined globally, the between-population effective contact rate $\left(\beta_{\text {bet }}\right)$ was specified as a proportion $(\eta)$ of the global within-population effective contact rate $\left(\beta_{\text {win }}\right)$. Variation in the between-population effective contact rate was modelled by selecting the values of $\beta_{\text {win }}$ from a Pert distribution. Thus, each iteration of the model used a unique value of $\beta_{\text {win }}$ selected from the distribution of values specified in Table 1 . The calculated number of transitions between the susceptible $(S)$ and exposed $(E)$ category resulting from inter-population contact were stochastically rounded to a whole number by comparison with a random number. For sub-population 1, the total number of transitions from the susceptible to exposed state per time step was calculated as

$$
\beta_{\text {win }}\left(\frac{S_{1} I_{1}}{N_{1}}\right)+\beta_{\text {bet }}\left(\frac{S_{1} I_{2}}{N_{1}+N_{2}}\right)+\beta_{\text {bet }}\left(\frac{S_{1} I_{3}}{N_{1}+N_{3}}\right)
$$

where subscripts denote sub-population number.

The model was validated by comparing the distribution of predicted average daily prevalences for the last 6 months of the iterations to results of sero-surveys conducted in pastoral areas of southern Sudan. The three population sizes were all set to 3333 to eliminate fade-out and to give a total population size (9999) that would permit comparison with the validation results of the single population SVEIQR model (Mariner et al., 2006). This simulation consisted of 2000 iterations to maximize the stability of estimates for the sensitivity analysis.

\subsection{Model experimentation}

Each simulation lasted 6 years. Sub-population 1 is treated as the reference subpopulation and all outcomes reported in this paper were effects on the reference subpopulation unless otherwise noted. The sub-populations 2 and 3 were denoted as contact populations. Sensitivity analysis was completed using multivariable linear regression and all coefficients were standardized.

The impact of population size and contact structure on the critical community size (CCS) required for the maintenance of infection was investigated first. The effect of decreasing sub-population sizes on the persistence of infection and the continuity of infection in the reference herd was investigated by successively reducing the size of the three sub-populations. The percentage of herds that were infected at the termination of the model, the percentage of herds continuously infected throughout the duration modeled and the mean number of days infected were determined. The impact of reducing the size of the reference sub-population while maintaining the size of the contact sub-populations at 500 was also investigated. The effect of varying the ratio $\eta$ of $\beta_{\text {bet }}$ to $\beta_{\text {win }}$ on the persistence of 
Table 1

Parameter definitions and default values for a spatially heterogeneous SVEIQR model of contagious bovine pleuropneumonia transmission in pastoral communities of East Africa ${ }^{\mathrm{a}}$

\begin{tabular}{|c|c|c|c|c|}
\hline Parameter & Definition & Min & Mode & Max \\
\hline$A$ & Amplitude of seasonal forcing ${ }^{c}$ & & 0.5 & \\
\hline$\alpha_{\mathrm{q}}$ & Rate of sequestrum formation & 0.011 & 0.013 & 0.018 \\
\hline$\alpha_{\mathrm{r}}$ & Rate of recovery & 0.0036 & 0.0045 & 0.0059 \\
\hline$\beta_{\text {win }}$ & Effective within group contact rate ${ }^{\mathrm{b}}$ & 0.07 & 0.126 & 0.127 \\
\hline$\beta_{\text {bet }}$ & Effective between-group contact rate & & $\eta \beta_{\text {win }}$ & \\
\hline E & Initial prevalence of exposed ${ }^{c}$ & 0.01 & 0.02 & 0.03 \\
\hline$\varepsilon$ & Vaccination efficiency ${ }^{\mathrm{c}}$ & & 0.8 & \\
\hline$\eta$ & Ratio of between to within group contact ${ }^{\mathrm{c}}$ & & 0.1 & \\
\hline$\gamma$ & Transition rate from exposed to infectious & 0.018 & 0.024 & 0.036 \\
\hline$I$ & Initial prevalence of infectious ${ }^{c}$ & 0.01 & 0.02 & 0.03 \\
\hline$\kappa$ & Rate of sequestrum reactivation & 0.00007 & 0.00009 & 0.00011 \\
\hline$\mu$ & Non-specific mortality rate & 0.00049 & 0.00055 & 0.00062 \\
\hline$N$ & Sub-population size & & 500 & \\
\hline$p_{\mathrm{e}}$ & Vaccine efficacy $^{c}$ & 0.5 & 0.65 & 0.8 \\
\hline$p_{\mathrm{v}}$ & Proportion vaccinated $^{c}$ & & 0.8 & \\
\hline$R$ & Initial prevalence of exposed ${ }^{c}$ & 0.50 & 0.55 & 0.60 \\
\hline$\rho$ & Proportion immunized $^{\mathrm{c}}$ & & $p_{\mathrm{v}} p_{\mathrm{e}} \varepsilon$ & \\
\hline$\sigma$ & CBPP specific mortality rate & 0.0064 & 0.009 & 0.013 \\
\hline$\omega_{\mathrm{n}}$ & Rate of loss of natural immunity & & 0.00027 & \\
\hline$\omega_{\mathrm{v}}$ & Rate of loss of vaccinal immunity & 0.00078 & 0.00091 & 0.0011 \\
\hline$\psi$ & Rate of sequestrum resolution & 0.0068 & 0.0075 & 0.0079 \\
\hline
\end{tabular}

${ }^{\mathrm{a}}$ All units are days ${ }^{-1}$ unless otherwise defined.

b The effective within group contact rate $(\beta)$ was equal to the physical contact rate $(C)$ multiplied by the probability of transmission per contact $(p)$ within sub-populations.

c Proportion.

infection was studied for a range of values between 0 and 0.1 over herd sizes of 50 to 500 head.

In regard to control options, treatment, vaccination, elective vaccination and combined vaccination and treatment scenarios were examined using an $\eta$ of 0.05 estimated from the data on community contact structure. The potential benefits of treatment were assessed by decreasing the infectious period and observing the effects on the persistence of infection and total mortality. The sum of $1 / \alpha_{\mathrm{r}}$ and $1 / \alpha_{\mathrm{q}}$ was reduced by increments of $25 \%$ while maintaining the proportionality of these 2 rates. The potential negative effect of treatment on the resistance of the persistently infected to infectious relapse was investigated by sequential doubling of the rate of sequestra reactivation, $\kappa$, in a series of simulations while maintaining the sum of $1 / \alpha_{\mathrm{r}}$ and $1 / \alpha_{\mathrm{q}}$ at $75 \%$ of their baseline value.

The impact of various pulsed mass annual and biannual vaccination programmes applied simultaneously to all three populations on the same day was investigated at three ranges of vaccine efficacy: 0.5 to $0.8,0.6$ to 0.8 and 0.7 to 0.8 . The proportion vaccinated and efficiency of vaccination were set to 0.8 in all mass vaccination simulations based on monitoring results of vaccination campaigns (IAEA, 1993). The impact of a simultaneous application of a moderately efficacious treatment programme $(1 / \alpha$ reduced by $25 \%)$ and a subset of vaccination strategies (vaccine efficacy of $0.5-0.8$ ) was modelled. 
Elective vaccination creates situations where individuals may choose to purchase vaccination for their own herd and other community members do not. In order to explore the impact of infection in non-vaccinated herds on the vaccinated herd, this was modelled as vaccination of the reference population in the absence of any systematic intervention in the 2 contact populations. A vaccine efficacy of $0.5-0.8$ was utilized. As payment for vaccination usually increases the accountability of service providers to clients, vaccination efficiency and coverage were set to 0.9 for elective vaccination simulations.

\section{Results}

The pathways of between cattle contact in the transhumant cattle keeping systems studied in southern Sudan were diverse both in terms of type and intensity. Contact may be grouped according to practices that affect mixing rates and practices that result in temporary or permanent transfer of animals between herds.

Seasonal patterns of transhumance were a major determinant of mixing patterns. The Jie, Murle and Kachipo practiced seasonal transhumance while the Dinka community in Boma maintained one large night enclosure year-round and the cattle were taken out to graze as one group.

The Boma area is dominated by large flood plain broken by occasional hills. To the East there is a hilly upland area and a major seasonal river, the Kengen, flows through the centre of the plain. The Jie, Murle and Kachipo maintained permanent villages in the hill and upland areas where the entire herd gathered for the rainy season. In the dry season, the majority of the herd descended to the plain and were kept in mobile cattle camps. Part of the community remained behind in the village, especially the very old and young, and a few animals in milk were left behind for their benefit. Camps were usually organized along clan lines, but one or more clans from the same or different tribes often occupied immediately adjacent areas.

Cattle traveled to and from dry season grazing areas throughout the dry season and the camps moved every few days to weeks following the retreating flood waters. The objectives were to manage the milk supply and maximize forage utilization while reducing exposure to disease vectors, other environmental risk factors and the risk of stock theft. Dry season grazing camps for the Jie and Murle consisted of a number of herds kept in individual thorn brush corrals. Smaller camps often fused or a large camp split depending on the availability of resources and the security situation. Although movement was a risk management tool for vector-borne and parasitic diseases, the pastoralists recognized that movement and mixing increased the risk for contagious disease, particularly CBPP. The dry season contact pattern could be summarized as a mosaic of camps shifting in spatial terms linked through a resilient framework of social relationships.

Mixing occurred in grazing camps, on pasture and at watering places. Jie, Murle and Kachipo pastoralists kept their herds in separate night enclosures separated by 50 to several hundred meters. Animals were not tethered, but calves on milk were kept in small enclosures within or adjacent to the principal coral. Herds were generally between 30 and 100 head. Dinka grouped all herds in contiguous area with each animal tethered to a peg. A Dinka cattle camp at Boma consisted of approximately 400 head. 
A number of forms of social exchange were identified that resulted in transfer of animals between herds. These included bride wealth payments, loans from wealthy to poor households, traditional legal settlements, and exchanges to bind friendships or as a risk avoidance mechanism. Cattle transfers such as bride wealth payments were usually subdivided and distributed over several households according to kinship ties. This form of exchange was important enough that having more sons than daughters was considered a principal predictor of poverty as net cattle flow would be out of the herd. Respondents estimated that they received cattle into their herd through traditional exchanges 6-10 times per year. These peaceful forms of social exchange occurred both within and between communities.

Cattle raiding was noted to be an additional major exchange pathway at the intercommunity level between competing groups. Raids and counter-raids frequently resulted in the re-distribution of thousands of head of cattle within months. A Venn diagram prepared by Jie pastoralists illustrating patterns of cattle exchange with neighbouring communities is presented in Fig. 1. Probing questions relative to the diagramming indicated that the Jie played a pivotal role in exchange with the Toposa, Kachipo and Murle communities and that the Toposa played a central role in regard to cattle raiding. These findings were confirmed in interviews with other communities and in proportional piling and participatory mapping exercises.

Turning to the results of the model experimentation, the distribution of the average daily prevalence of infection for the final 6 months of the simulation is presented in Fig. 2. The mean prevalence was $6.4 \%$ with a range of $2.1-10.6 \%$. The animal level prevalence of infection was most sensitive to the CBPP specific mortality rate (Fig. 3) with a standardized regression coefficient of -0.37 . This signified that 1 standard deviation change in the value

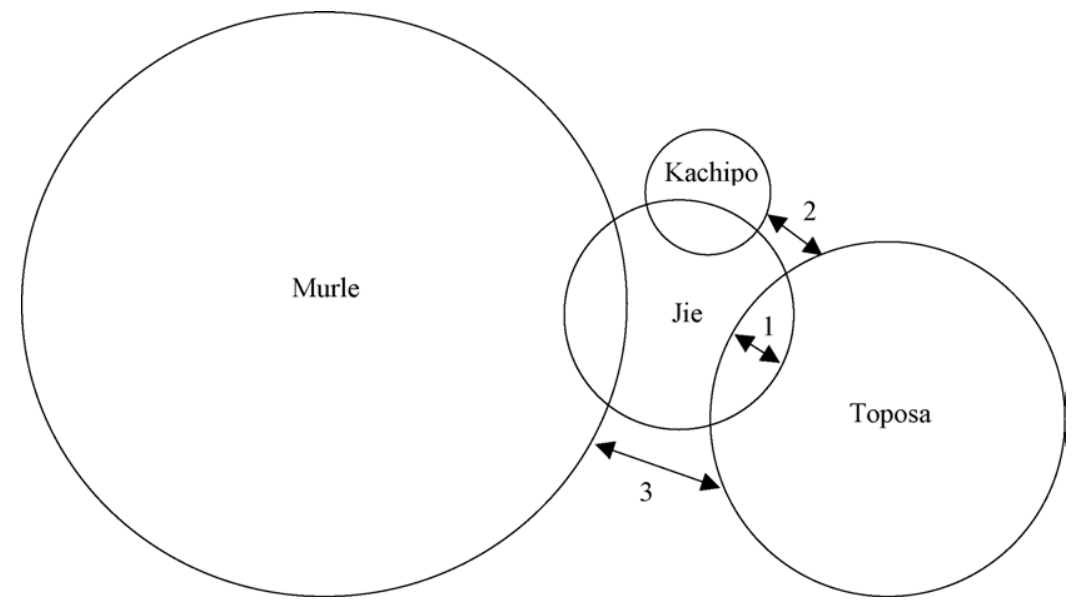

Fig. 1. The interactions between the Jie and the Murle, Toposa and Kachipo peoples as perceived by the Jie. Overlap of the circles indicated contact through herding practices, exchange or loan of livestock and payment of bride wealth. The arrows indicated cattle raiding pathways and the numbers represented the ranking assigned by the Jie in terms of the size of the problem. Note that the respondents used double headed arrows to indicate cattle raiding as cattle raids were usually followed by counter-raids. The diagram indicated that the Jie were a key link between all groups in terms of peaceful social exchange and that the Toposa played a central role in cattle raiding. 


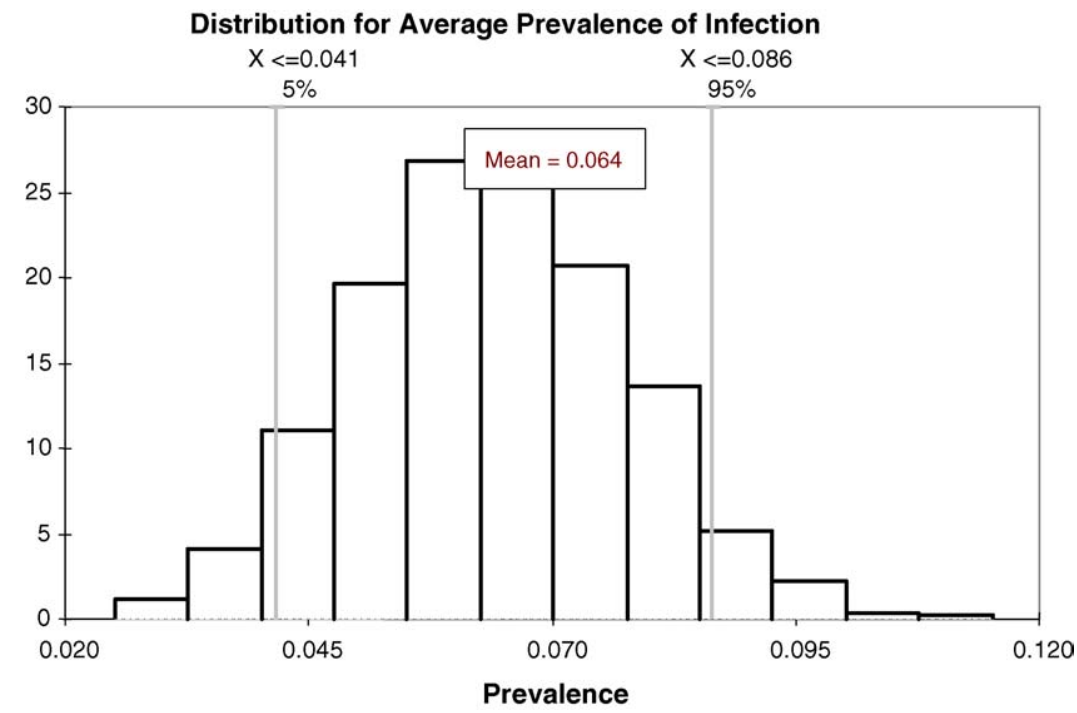

Fig. 2. Distribution of the average daily prevalence of infection over the last 6 months of each iteration of the model. The simulation was based on three equal populations of 3333 head and did not incorporate seasonal forcing. The mean prevalence was $6.4 \%$ and $90 \%$ of the iterations ended with prevalence between 4.1 and $8.6 \%$.

\section{Regression Sensitivity for Average Prevalence of Infection}

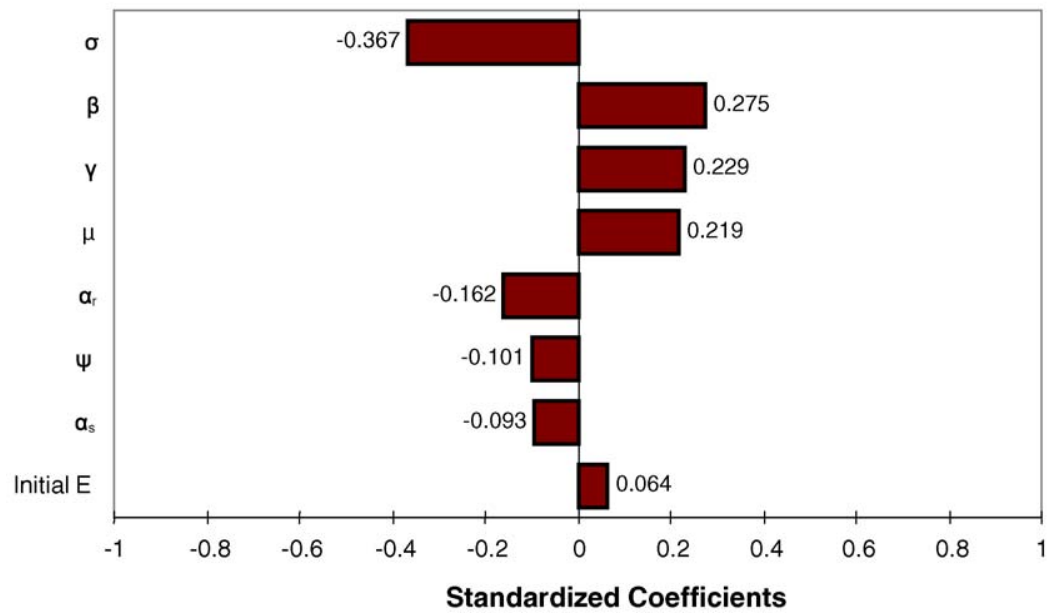

Fig. 3. Plot of the sensitivity analysis for prevalence of infection. Note that the prevalence of infection (infectious and persistently infected animals) was most sensitive to the CBPP specific mortality rate $(\sigma)$, followed by the latent period $(\gamma)$ and the rate of sequestra formation $\left(\alpha_{\mathrm{s}}\right)$. The $r^{2}$ for the regression model was 0.3842 for this simulation of 2,000 iterations using the default parameter estimates. 
of $\sigma$ corresponded to a change of -0.37 standard deviations in the average daily prevalence. The value of $r^{2}$ for the sensitivity analysis was 0.38 . The average case mortality proportion was $33.2 \%$ with a range of $22.6-50 \%$. The average prevalence of persistently infected animals with infected sequestra was predicted by the model to be $4.0 \%$ with a range of $1.6-7.4 \%$.

With regard to the effects of population and contact structure, the effect of decreasing population size on the prevalence of infected herds in the overall population and continuity of infection in the reference population is shown in Table 2. With population sizes of 500 head, $73 \%$ of the reference populations remained continuously infected throughout the 6-year duration of the simulation. A total population of 120 head (madeup of 3 sub-populations of 40 head) was still capable of maintaining infection $(0.2 \%)$. Below a sub-population size of 75 , the reference sub-population could not support continuous infection.

When the size of the reference sub-population was reduced to 50 head and the size of the contact sub-populations was maintained at 500 head, $16.2 \%$ of the reference herds were still infected at the end of the simulation but only $1.8 \%$ of the herds had been infected continuously. Observation of the epidemic curves during the simulation indicated most reference herds experienced fade-out at least once, but that they were re-infected by the contact herds 1 or more times.

The results of varying the value of $\eta$ in simulations with 3 herds of equal size are shown in Table 3. Note that inclusion of even a very low value of $\eta$ of 0.0005 decreased the CCS capable of supporting infection by about 100 head and increased the persistence in herds of 500 head by $54.7 \%$ when compared with simulations with no between-group contact $(\eta=0)$. An $\eta$ value of 0.005 allowed disease to persist in $46.0 \%$ of herds of 500 head. This was equivalent to more than $54.4 \%$ of the effect of the highest between herd contact ratios examined (0.1).

The results of decreasing the infectious period are shown in Table 4 . Reducing the infectious period by $50 \%$ caused a $64 \%$ reduction in mortality and increased the rate of

Table 2

Persistence of contagious bovine pleuropneumonia (CBPP) infection as a function of herd size in the spatially heterogeneous SVEIQR model of CBPP transmission in pastoral communities of East Africa

\begin{tabular}{llcc}
\hline Herd size $^{\mathrm{a}}$ & $\begin{array}{l}\text { Herd prevalence at } \\
\text { termination of simulation }\end{array}$ & Mean days infected & Percent continuously $_{\text {infected }^{\mathrm{c}}}$ \\
\hline 500 & 75.4 & 1945 & 73.0 \\
400 & 59.0 & 1846 & 58.8 \\
300 & 38.6 & 1621 & 37.8 \\
200 & 23.0 & 1432 & 18.0 \\
100 & 5.2 & 1064 & 3.2 \\
75 & 3.0 & 911 & 1.8 \\
50 & 0.4 & 727 & 0.0 \\
40 & 0.2 & 599 & 0.0 \\
30 & 0.0 & 476 & 0.0 \\
\hline
\end{tabular}

\footnotetext{
${ }^{a}$ Individual herd size for each of the thre herds of equal size.

${ }^{\mathrm{b}}$ Over the 2141 day ( $~ 6$ year) duration of the model.

${ }^{\mathrm{c}}$ Herds infected at least 2140 days.
} 
Table 3

The end-stage herd prevalence as a function of between-herd contact and herd size in the spatially heterogeneous SVEIQR model of contagious bovine pleuropneumonia transmission in pastoral communities of East Africa

\begin{tabular}{lllllll}
\hline Herd size $^{\mathrm{a}}$ & \multicolumn{5}{l}{ Ratio $(\eta)$ of $\beta_{\text {bet }}$ to $\beta_{\text {win }}$} \\
\cline { 2 - 7 } & 0 & 0.0005 & 0.005 & 0.025 & 0.05 & 0.1 \\
\hline 500 & 10.6 & 16.4 & 46.0 & 65.6 & 75.4 & 84.4 \\
400 & 5.4 & 7.6 & 32.2 & 51.0 & 59.0 & 72.8 \\
300 & 1.0 & 3.0 & 14.0 & 35.6 & 38.6 & 52.4 \\
200 & 0.2 & 0.2 & 5.2 & 12.6 & 23.0 & 30.8 \\
100 & 0.0 & 0.2 & 1.2 & 2.8 & 5.2 & 8.0 \\
75 & 0.0 & 0.0 & 0.6 & 1.2 & 3.0 & 5.0 \\
50 & 0.0 & 0.0 & 0.2 & 0.2 & 0.4 & 0.6 \\
\hline
\end{tabular}

a Three herds, each of equal size.

Table 4

The effect of reducing the duration of the infectious period from 56 to 28 days on the persistence of infection in the spatially heterogeneous SVEIQR model of contagious bovine pleuropneumonia transmission in pastoral communities of East Africa modelled as three herds of 500 head

\begin{tabular}{lllllllll}
\hline $\begin{array}{l}\text { Duration } \\
(1 / \alpha)\end{array}$ & $R_{0}{ }^{\mathrm{a}}$ & $\begin{array}{l}\text { Herd } \\
\text { prevalence } \\
(\%)^{\mathrm{b}}\end{array}$ & $\begin{array}{l}\text { Mean } \\
\text { duration of } \\
\text { infection }^{\mathrm{c}}\end{array}$ & $\begin{array}{l}\text { Case } \\
\text { fatality } \\
\text { proportion }^{2}\end{array}$ & $\begin{array}{l}\text { Case } \\
\text { sequestration } \\
\text { proportion }^{\mathrm{d}}\end{array}$ & $\begin{array}{l}\text { Mean } \\
\text { total } \\
\text { cases }\end{array}$ & $\begin{array}{l}\text { Mean } \\
\text { total } \\
\text { sequestra }^{\mathrm{e}}\end{array}$ & $\begin{array}{l}\text { Mean } \\
\text { total } \\
\text { mortality }^{2}\end{array}$ \\
\hline 56 & 4.10 & 75.4 & 1945 & 0.33 & 0.49 & 602 & 293 & 178 \\
42 & 3.33 & 59.6 & 1791 & 0.28 & 0.54 & 469 & 251 & 129 \\
28 & 2.37 & 33.2 & 1475 & 0.20 & 0.58 & 320 & 185 & 64 \\
\hline
\end{tabular}

${ }^{a}$ The values of $R_{0}$ presented consider only transmission within the reference population (Mariner et al., 2006).

b The percentage of reference herds that had at least 1 infected (infectious or persistently infected) animal at the end of the 6 year modelling period.

${ }^{c}$ Note that in herds where the disease did not fade-out, the duration was truncated at 2141 days ( $\sim 6$ years).

d The proportion of infectious cases that evolved to the persistently infected state.

e The mean total number of persistently infected animals that developed in the reference herd of 500 head over the 6 year duration of the model.

fade-out. The prevalence of infected herds was reduced from 75.4 to $33.2 \%$. As duration of the infectious period was reduced, the case fatality proportion decreased, the case sequestration proportion increased and the mean number of sequestra formed decreased. In the simulations where the rate of sequestra reactivation was sequentially doubled, the rate of sequestrum reactivation had to be increased eight-fold to return the prevalence of infected herds $(73.6 \%)$ to the same level as those found in scenarios using the baseline estimates of $\alpha(75.4 \%)$. Even when $\kappa$ was increased 16-fold, positive benefits in terms of an overall mortality reduction of $7.3 \%$ were still observed.

The results of simulations under various vaccination regimes are described in Table 5. A 5 year annual pulse mass vaccination programme using a vaccine with an efficacy of 0.5 0.8 reduced the total mean mortality by $27.0 \%$ in the reference population. The prevalence of infected herds at the end of the period modelled was reduced from $75.4 \%$ when there was no intervention to $67.8 \%$. A 5 year biannual programme of pulse vaccination with a vaccine of similar quality reduced mortality by $53.4 \%$ and left $35.2 \%$ of the herds infected. 
Table 5

The effect of vaccination on the end-stage herd prevalence, duration of infection and mortality in the spatially heterogeneous SVEIQR model of contagious bovine pleuropneumonia transmission in pastoral communities of East Africa modelled as three herds of 500 head

\begin{tabular}{|c|c|c|c|c|}
\hline $\begin{array}{l}\text { Vaccination } \\
\text { programme }^{\mathrm{a}}\end{array}$ & $\begin{array}{l}\text { Vaccine } \\
\text { efficacy }(\varepsilon)\end{array}$ & $\begin{array}{l}\text { Herd } \\
\text { prevalence }(\%)\end{array}$ & $\begin{array}{l}\text { Mean duration } \\
\text { of infection }^{b}\end{array}$ & $\begin{array}{l}\text { Mean total } \\
\text { mortality }\end{array}$ \\
\hline No vaccination & - & 75.4 & 1945 & 178 \\
\hline Annual - 5 year & $0.5-0.8$ & 67.8 & 1893 & 130 \\
\hline Biannual - 2 year & & 57.2 & 1761 & 129 \\
\hline Biannual - 3 year & & 48.0 & 1567 & 106 \\
\hline Biannual - 4 year & & 41.0 & 1498 & 93 \\
\hline Biannual - 5 year & & 35.2 & 1492 & 83 \\
\hline Annual - 5 year & $0.6-0.8$ & 68.2 & 1897 & 127 \\
\hline Biannual - 5 year & & 28.0 & 1411 & 74 \\
\hline Biannual - 5 year & $0.7-0.8$ & 21.2 & 1323 & 66 \\
\hline
\end{tabular}

\footnotetext{
${ }^{\text {a }}$ Frequency and duration of campaign.

b Note that in the reference herds of 500 head where the disease did not fade-out, the duration was truncated at 2141 days $(\sim 6$ years $)$.
}

Increasing vaccine efficacy to a range of $0.7-0.8$, reduced mortality by $62.9 \%$ and left $21.2 \%$ of the reference herds infected.

As shown in Table 6, elective vaccination had almost no impact on the prevalence of infected herds at the termination of the model. However, the number of cases within herds was reduced and an important impact on mean total mortality was observed. A 5 year annual programme resulted in a $30.3 \%$ reduction in mortality. Vaccination did not make a major contribution in terms of population-wide disease eradication through elimination of infected herds, but did lead to important within herd reductions in mortality that would be of benefit to individual farmers.

Table 6

The impact of elective vaccination on disease persistence and mortality in the spatially heterogeneous SVEIQR model of contagious bovine pleuropneumonia transmission in pastoral communities of East Africa modelled as three herds of 500 head $^{\mathrm{a}}$

\begin{tabular}{llll}
\hline Vaccination programme $^{\mathrm{b}}$ & ${\text { Herd prevalence }(\%)^{\mathrm{c}}}$ & Mean duration of infection $^{\mathrm{d}}$ & Mean total mortality \\
\hline No vaccination & 75.4 & 1945 & 178 \\
Annual - 5 year & 71.8 & 1905 & 124 \\
Biannual - 2 year & 69.8 & 1886 & 136 \\
Biannual - 3 year & 68.6 & 1822 & 121 \\
Biannual - 4 year & 67.6 & 1812 & 109 \\
\hline
\end{tabular}

\footnotetext{
${ }^{\mathrm{a}}$ Proportional vaccine efficacy $=0.5-0.8$, proportion vaccinated $=0.9$, vaccination efficiency $=0.9$.

b Frequency and duration of campaigns.

c The percent of reference herds that had at least 1 infected (infectious or persistently infected) animal at the end of the 6 year simulation.

${ }^{\mathrm{d}}$ Note that in the reference herds of 500 head where the disease did not fade-out, the duration was truncated at 2141 days ( $\sim 6$ years).
} 
Table 7

The effect of simultaneous vaccination and reductions in the infectious period $(1 / \alpha)$ on persistence of infection and mortality in the spatially heterogeneous SVEIQR model of contagious bovine pleuropneumonia transmission in pastoral communities of East Africa modelled as three herds of 500 head $^{\mathrm{a}}$

\begin{tabular}{|c|c|c|c|c|c|c|}
\hline \multirow[t]{2}{*}{ Vaccination programme ${ }^{b}$} & \multicolumn{2}{|l|}{ Baseline $1 / \alpha$} & \multicolumn{2}{|l|}{$75 \% 1 / \alpha$} & \multicolumn{2}{|l|}{$50 \% 1 / \alpha$} \\
\hline & Prevalence ${ }^{c}$ & Mortality & Prevalence & Mortality & Prevalence & Mortality \\
\hline None & 75.4 & 178 & 59.6 & 129 & 33.2 & 64 \\
\hline Annual - 5 year & 67.8 & 130 & 43.0 & 79 & 7.6 & 24 \\
\hline Biannual - 2 year & 57.2 & 129 & - & - & 4.4 & 20 \\
\hline Biannual - 3 year & 48.0 & 106 & - & - & 1.6 & 17 \\
\hline Biannual - 5 year & 35.2 & 83 & 8.0 & 43 & 0.4 & 16 \\
\hline
\end{tabular}

\footnotetext{
${ }^{\text {a }}$ Proportional vaccine efficacy $=0.5-0.8$, proportion vaccinated $=0.8$, vaccination efficiency $=0.8$

b Frequency and duration of campaigns.

c The percent of reference herds that had at least 1 infected (infectious or persistently infected) animal at the end of the 6 year simulation.
}

The impact of four vaccination scenarios in the presence and absence of a reduced infectious period are presented in Table 7. In a 5 year programme of annual vaccination, a $25 \%$ reduction of the infectious period decreased the prevalence of infection by an additional $36.6 \%$ and a $50 \%$ reduction in infectious period reduced the herd level prevalence by $60.2 \%$ relative to the same vaccination programme using baseline estimates for the recovery rates. When a 5 year programme of biannual vaccination was combined with a 50\% reduction of the length of the infectious period, the prevalence of infected herds was reduced to $0.4 \%$.

\section{Discussion}

The key issues in contact structure are the size of groups, the relations between groups, and the resulting variation in contact rates, prevalence and persistence of infection.

In traditional communities, the structure and customary relations of human populations is the primary determinant of livestock population dynamics. Pastoral cattle populations are highly structured along herd, dry season grazing camp, wet season settlement, clan and tribal lines. Within local and allied communities, between-herd contact rates are high but have a pronounced seasonal fluctuation. At higher levels of community structure, clan and tribal contact patterns are complex and temporally dynamic. At these higher levels of aggregation, contact between neighboring groups can range from intensive to non-existent.

The finding that cattle exchange between herds and community groups is an important part of the pastoral economy is well supported in the literature (Niamir-Fuller, 1999). A pastoral herd is a group of animals that are managed on a day to day basis by 1 family but due to the complex web of cattle ownership and use rights, the pastoral herd is a fluid entity. Cattle exchange is of particular interest in the epidemiology of diseases with long infected and infectious periods.

Super-imposed upon these positive social exchanges is the problem of cattle raiding that occurs throughout much of East Africa. This form of exchange is especially pertinent to the epidemiology of CBPP. A number of Kurya respondents and local veterinary staff of the 
Mara region of Tanzania attributed the re-introduction of CBPP into the Mara area to cattle raiding between 1985-87 (Mariner et al., 2003). The literature also describes the importance of cattle raiding in the spread of CBPP within the Mara region of Tanzania (Masiga et al., 1996). Cross border raiding, is a major concern in the Karimajong cluster area of Kenya, Uganda, Sudan and Ethiopia (Niamir-Fuller, 1999) as well as the for the Somali ecosystem of northeastern Kenya, southern Ethiopia and Somalia. At times, larger political conflicts drive raiding and lead to large scale migrations of people and livestock or complete loss of livestock by an entire community.

Thus, a model of contagious disease transmission in pastoral communities that is structured and allows high between-herd contact will more accurately reflect the system. All things considered, scaling the between-herd effective contact rate at $5 \%$ of the within herd effective contact rate may have been conservative. Our approach of dividing $\beta$ into components was appropriate for representing between herd mixing but should only be considered an approximation for temporary and permanent exchange of animals. In addition, adding between-herd contact as a percentage of with-in herd contact results in a slight increase the overall $\beta$ of the heterogeneous system relative to the homogeneous model. A more realistic (and complex) method of modelling between-herd exchanges would be to build in animal transfers between sub-populations.

The simulations at various herd sizes and inter-group contact rates indicated that pastoral herd structure and contact patterns decrease the CCS required for the persistence of CBPP. In the heterogeneous model with individual herds of 50 head (a total population of 150 head), $1.8 \%$ of the reference herds were infected at the termination of the simulation suggesting a CCS of 150 head. This should be compared with the CCS of 300 head found in the homogeneous SVEIQR model (Mariner et al., 2006). The heterogeneous model suggests small pastoral communities with a few herds of modest size are capable of maintaining infection indefinitely. This is perhaps the single most pertinent concern in regard to CBPP control in pastoral areas.

The heterogeneous model was much more resistant to disease fade-out than the homogeneous population model. With herd sizes of 500 head, $75.4 \%$ of herds were infected at the termination of the simulation, whereas in the homogeneous model with all the same parameter settings, a mean herd prevalence of only $10.6 \%$ was found at the end of the 6 year simulation period (Mariner et al., 2006). This is in agreement with the more general analytical finding that $R_{0}$ calculated treating a population as homogeneous is equal to or less than $R_{0}$ calculated treating the population as a group of inter-liked subpopulations (Adler, 1992; Diekmann and Heesterbeek, 2000).

The results and observation of the herd-level epidemic curves generated by the model indicated that fade-out among the three herds of equal size was a synchronized event. Of the $75.4 \%$ of herds that were infected at the end of the 500 head simulation $73.0 \%$ were continuously infected. This difference of $2.4 \%$ is indicative of a small number of iterations where the reference herd was re-infected by 1 of the contact herds.

When the herd size of the reference herd was reduced to 50 head and the size of the contact herds was maintained at 500 head, the final herd prevalence of infection was $16.2 \%$ but only $1.8 \%$ had been continuously infected. This means the disease faded-out at least once from all but $1.8 \%$ of the reference herds but was reintroduced and remained present at the end of the model run in an additional $14.4 \%$ of herds. This suggests that the efficiency 
of disease control interventions will be increased if the concept of CCS is exploited and interventions are focused on the larger herds within a community.

The mean animal level prevalences of infection predicted by the heterogeneous and homogeneous SVEIQR models were 6.5 and 5.9\%, respectively (Mariner et al., 2005). In CBPP, seroprevalence is an sensitive indicator of current infection status and not history of exposure (Windsor, 1977). Zessin et al. (1985) measured a seroprevalence of $8.1 \%$ in Dinka cattle and 9.2\% in Fulani herds the Bahr el Ghazal. McDermott et al. (1987) found a seroprevalence of $8.1 \%$ in Dinka cattle in Kongor. As part of this study, a seroprevalence of 4.7\% was measured in the Boma area in Jie and Murle herds. The high prevalence Dinka and Fulani communities both noted that mortality was sporadic (Zessin et al., 1985; McDermott et al., 1987), whereas the lower prevalence Jie and Murle communities consistently reported large clinical case mortality proportions. The sensitivity of prevalence estimates obtained from the model to the case fatality proportion and field observations suggest that the differences between the observed prevalence of infection in sero-surveys may be due in part to an inverse relationship between case mortality proportion and prevalence of infection.

This result has important implications for the design of surveillance programmes and assessments of CBPP impact. It suggests that seroprevalence data alone are insufficient to assess impact as severity and serologic prevalence are inversely related. Low prevalence estimates in the absence of other data can in fact mask high total mortality and other important disease impacts (Rothman and Greenland, 1998). The authors suggest that CBPP serology should constitute only one component of a broader participatory impact assessment process.

The ultimate goal of this paper was to identify realistic CBPP control objectives and strategies for achieving those objectives. The sensitivity of disease prevalence to the mortality proportion supports the key role that stamping out has played in the history of CBPP control. The model highlights the theoretical utility of historical quarantine and stamping-out methods of CBPP control. The homogenous model found a much larger CCS for the maintenance of infection than the heterogeneous model. This emphasizes the potential effectiveness of movement control and quarantine even in the absence of other interventions. However, the sensitivity analysis conducted on $\eta$ indicated that even small amounts of between-herd contact dramatically increased the persistence of CBPP. This suggests that achievable levels of movement control and quarantine attainable in East Africa today are unlikely to contribute CBPP control within currently affected areas. Realistically, movement control and stamping out are not viable in sub-Saharan Africa on the scale required for CBPP eradication with existing vaccines.

Turning to mass vaccination, the introduction of spatial heterogeneity into the model reduced the impact of mass vaccination as measured in the homogeneous model. This result is in agreement with May and Anderson (1984) who found that higher blanket immunization rates were required to eradicate disease in structured populations and they recommended a focused approach where high risk (larger) population groups were specifically targeted in order to increase efficiency.

The analysis suggested that even heroic mass vaccination programmes in pastoral areas are unlikely to have significant impact on the prevalence of CBPP infected herds. These results are born out by field observations where both authorities and livestock owners reported limited benefits from vaccination (Mariner et al., 2003). And, the analysis does not 
incorporate the localized but often severe reactions and mortality resulting from vaccination with the T1/44 strain (Thiaucourt, 2002). Beyond the economic costs of vaccine induced reactions, these effects preclude the high coverage required to generate impact with vaccines of moderate efficacy and duration of immunity. Finally, the present era of declining public sector funding for animal health interventions makes sustained, intensive campaigns an unrealistic strategy. This all suggests that public veterinary services might be better advised to contemplate more achievable objectives than population level suppression of CBPP through mass vaccination.

The options that remain are herd level control of CBPP control either through elective vaccination, treatment or a combined program of elective vaccination of healthy animals and treatment of apparent clinical cases. The modelling results for all three options suggested important benefits to herd owners from all three approaches.

The model demonstrated that elective vaccination practiced in only one of three herds had almost no impact on the prevalence of infected herds even among vaccinated herds. However, the herd owner was able to capture essentially all of the benefit of vaccination in terms of reduction of the number of cases and death losses. Assuming a high cost of 1 USD per vaccination and a conservative value of 100 USD per head of cattle, the livestock owner would avoid roughly 2 USD of death loss for every 1 USD spent on an annual vaccination programme.

The effect of reducing the infectious period by $50 \%$ had greater impact on mortality than any of the vaccination regimes modelled. The effect on the prevalence of infected herds was as great as the vaccination schemes that assumed a proportional vaccine efficacy of $0.6-0.8$. Effective treatment schemes are equivalent to the reduction of the infectious period leading to a reduction in the effective reproductive number. The proportion of cases that resolved as sequestra increased as more animals survived due to the shorter duration of the clinical period. However, the mean number of sequestra formed declined as the incidence of new cases was reduced and there were fewer cases overall to develop sequestra. An effective treatment regime will reduce mortality and the number of persistently infected animals.

It has been argued (without empirical evidence) that treatment leads to the formation of sequestra that are qualitatively different and more prone to reactivation. Dramatic increases of the value of $\kappa$, the rate of sequestra re-activation, were required to reverse the positive impact of treatment on the persistence and prevalence of infected herds. The potential benefits of treatment in terms of mortality reduction were even more resistant to the impact of a hypothetical increase in the rate of sequestrum re-activation.

The model indicated that combining treatment of clinical cases with vaccination of healthy herd members can enhance control. A 5 year programme of biannual vaccination combined with a hypothetical $50 \%$ reduction of the infectious period due to treatment came the closest of any potential intervention to eradicating CBPP from a heterogeneous population of 3 herds of 500 head. Treatment is widely practiced in the field, but has never been properly researched. The potential impact of treatment and the acceptability of treatment by livestock owners strongly indicate that controlled trials should be undertaken without delay.

The three population heterogeneous SVEIQR model illustrates the impact of population structure on CBPP epidemiology and control when compared with a single homogenous population model. The model predicts that structured populations support an overall higher prevalence of disease and that disease is more difficult to eradicate when the population is 
structured. Considering that we have not explicitly modelled between-herd transfers of animals, our intervention impact predictions are probably still optimistic.

Our work has shown that eradication of CBPP with available tools under present socioeconomic conditions has a low probability of success. Comparison of the two models reveals that strong movement control is a prerequisite for disease eradication in mass vaccination campaigns with available vaccines. However, movement is key to pastoral livelihoods. Attempts at movement control over the past two decades have largely failed due social, economic and political forces that outweigh the benefits of CBPP control. We have shown that elective vaccination is a powerful tool for the economically-viable control of CBPP losses at the herd level. We have also demonstrated that effective treatment regimes have tremendous potential benefit. The constraints to capturing these benefits largely lie in government and international animal health policies that have not evolved in step with social, economic and political conditions.

Nominally, CBPP is a disease identified by most veterinary authorities for control by public mass vaccination campaigns with or without an element of cost recovery. As a result, vaccine is not available for private purchase on the regulated professional market. Due to budgetary and logistic constraints, public mass vaccinations campaigns are not carried out regularly. There is little hope that resources will be made available to national governments for open-ended commitment to institutionalized mass vaccination programmes. As a result of these policy mismatches, livestock owners cannot obtain CBPP vaccination and are unable to engage in effective elective control programmes.

The research indicates that a fundamental shift in CBPP control policies is required (AU/IBAR, 2002). A policy of regulated private control should replace public mass control. The authors recommend that the licensed CBPP vaccine be made available through regulated commercial channels. The objective is to empower private and community-based service providers to implement elective programmes of CBPP control in partnership with livestock owners. Veterinary services should focus their efforts on regulating supplies of vaccines and promoting best practices in effective herd-level control.

\section{Acknowledgement}

The authors would like to acknowledge the support of the Community-based Animal Health and Participatory Epidemiology (CAPE) Unit of the Pan African Programme for the Control of Epizootics (PACE), African Union/Interafrican Bureau for Animal Resources for their support of the fieldwork, initial modelling work and preparation of manuscripts. The CAPE Unit is funded by the Department for International Development (United Kingdom). The authors wish to thank the European Union, the PACE Epidemiology Unit and the Animal Health Division of FAO for their support of the main modelling study.

\section{References}

Adler, F.R., 1992. The effects of averaging on the basic reproductive ratio. Math Biosci. 111, 89-98.

AU/IBAR, 2002. Contagious bovine pleuropneumonia in pastoralist areas of East Africa: disease dynamics and control options. African Union/Interafrican Bureau for Animal Resources, Nairobi. 
Catley, A., Irungu, P., Simiyu, K., Dadye, J., Mwakio, W., Kiragu, J., Nyamwaro, S.O., 2002. Participatory investigations of bovine trypanosomiasis in Tana River District, Kenya. Med. Vet. Entomol. 16, 1-12.

Catley, A., Okoth, S., Osman, J., Fison, T., Njiru, Z., Mwangi, J., Jones, B.A., Leyland, T.J., 2001. Participatory diagnosis of a chronic wasting disease in cattle in southern Sudan. Prev. Vet. Med. 51, 161-181.

Diekmann, O., Heesterbeek, J.A.P., 2000. Mathematical Epidemiology of Infectious Diseases: model building, analysis and interpretation. John Wiley \& Sons, Chichester.

Hammond, J.A., Branagan, D., 1965. Contagious bovine pleuropneumonia in Tanganyika. Bull. Epizootic Dis. Africa 13, 121-147.

IAEA, 1993. The Sero-Monitoring of Rinderpest Throughout Africa: Phase II. FAO/IAEA Division of Nuclear Techniques in Food and Agriculture, International Atomic Energy Agency, Vienna.

Mariner, J.C., Aluma Araba, Makungu S., 2003. Consultancy on the Dynamics of CBPP Endemism and the Development of Effective Control/Eradication Strategies for Pastoral Communities: Final Data Collection Report. Nairobi, The Community Animal Health and Participatory Epidemiology Unit of the African Union Interafrican Bureau for Animal Resources.

Mariner, J.C., McDermott, J., Heesterbeek, J.A.P., Catley, A., Thomson, G., Martin, S.W., 2006. A model of contagious bovine pleuropneumonia transmission dynamics in East Africa. Prev. Vet. Med. 73, 55-74.

Mariner, J.C., Paskin, R., 2000. Manual on Participatory Epidemiology. Food and Agriculture Organisation, Rome, $81 \mathrm{pp}$.

Masiga, W.N., Domenech, J., Windsor, R.S., 1996. Manifestation and epidemiology of contagious bovine pleuropneumonia in Africa. Rev. Sci. Tech. 15, 1283-1308.

May, R.M., Anderson, R.M., 1984. Spatial heterogeneity and the design of immunization programmes. Math Biosci. 72, 83-111.

McDermott, J.J., Deng, K.A., Jayatileka, T.N., El Jack, M.A., 1987. A cross-sectional cattle disease study in Kongor Rural Council, southern Sudan. I. Prevalence estimates and age, sex and breed associations for brucellosis and contagious bovine pleuropneumonia. Prev. Vet. Med. 5, 111-123.

Niamir-Fuller, M., 1999. Conflict management and mobility among pastoralists in Karamoja, Uganda. In: NiamirFuller, M. (Ed.), Managing Mobility in African Rangelands. Intermediate Technology Publications, London, pp. 149-183.

Rothman, K.J., Greenland, S., 1998. Modern Epidemiology. Lippincott-Raven, Philadelphia, 738 pp.

Thiaucourt, F., 2002. Contagious bovine pleuropneumonia and vaccine strain T1/44. Vet. Rec. 151, 156.

Windsor, R.S., 1977. The diagnosis of contagious bovine pleuropneumonia. Vet. Annu. 17, 59-65.

Windsor, R.S., Masiga, W.N., 1977. Investigations into the role of carrier animals in the spread of contagious bovine pleuropneumonia. Res. Vet. Sci. 23, 224-229.

Zessin, K.H., Baumann, M., Schwabe, C.W., Thorburn, M., 1985. Analysis of baseline surveillance data on contagious bovine pleuropneumonia in the Southern Sudan. Prev. Vet. Med. 3, 371-389. 